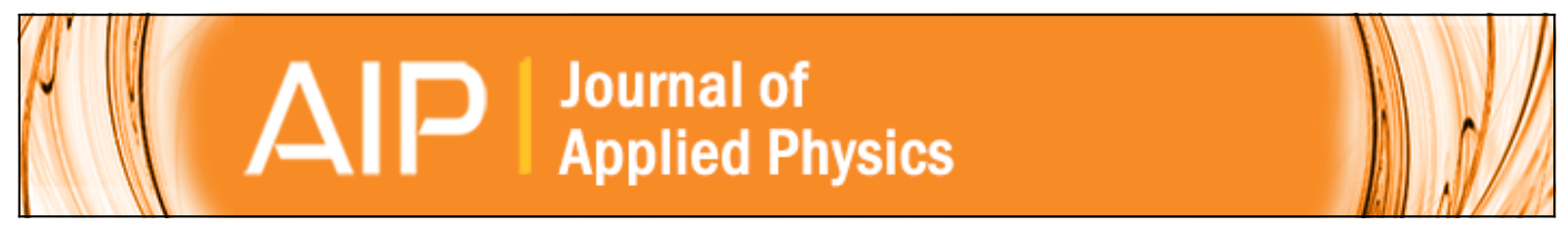

\title{
Influence of photon recycling on semiconductor luminescence refrigeration
}

J.-B. Wang, S. R. Johnson, D. Ding, S.-Q. Yu, and Y.-H. Zhang

Citation: Journal of Applied Physics 100, 043502 (2006); doi: 10.1063/1.2219323

View online: http://dx.doi.org/10.1063/1.2219323

View Table of Contents: http://scitation.aip.org/content/aip/journal/jap/100/4?ver=pdfcov

Published by the AIP Publishing

\section{Articles you may be interested in}

Multi-phonon-assisted absorption and emission in semiconductors and its potential for laser refrigeration Appl. Phys. Lett. 104, 221115 (2014); 10.1063/1.4880799

Photon recycling effect on electroluminescent refrigeration

J. Appl. Phys. 111, 014511 (2012); 10.1063/1.3676249

Thermal links for the implementation of an optical refrigerator

J. Appl. Phys. 105, 013116 (2009); 10.1063/1.3062522

Analysis of optothermionic refrigeration based on semiconductor heterojunction

J. Appl. Phys. 99, 074504 (2006); 10.1063/1.2188249

Cooling to $208 \mathrm{~K}$ by optical refrigeration

Appl. Phys. Lett. 86, 154107 (2005); 10.1063/1.1900951

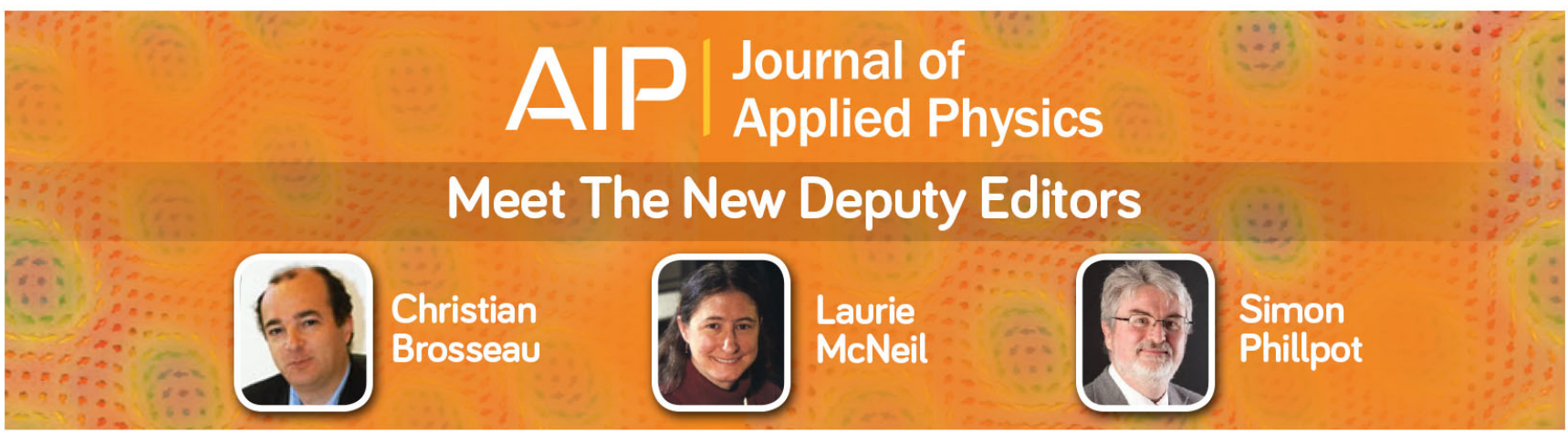




\title{
Influence of photon recycling on semiconductor luminescence refrigeration
}

\author{
J.-B. Wang, S. R. Johnson, D. Ding, S.-Q. Yu, and Y.-H. Zhang ${ }^{\text {a) }}$ \\ Center for Solid State Electronics Research, Department of Electrical Engineering, Arizona State \\ University, Tempe, Arizona 85287
}

(Received 14 December 2005; accepted 25 May 2006; published online 16 August 2006)

\begin{abstract}
Luminescence refrigeration in semiconductors is studied using a model that includes the rate equations for carriers and photons as well as the influence of spectral dependent photon recycling. Expressions are derived for cooling efficiency, cooling power density, and the minimum external quantum efficiency required for cooling. These results show that net cooling is accessible and that photon recycling significantly contributes to luminescence refrigeration when the luminescence extraction is small. (C) 2006 American Institute of Physics. [DOI: 10.1063/1.2219323]
\end{abstract}

\section{INTRODUCTION}

An optical cooler has recently been demonstrated using photoluminescence upconversion in doped glass pumped by a solid-state laser. ${ }^{1,2}$ Unlike thermoelectric coolers in which heat is transferred from the hot to the cold side of the device, luminescence based coolers can effectively transport heat from the device into free space. Furthermore, luminescence coolers are expected to have a higher cooling efficiency at low temperatures and a broader range of operating temperatures. Since it is difficult to monolithically integrate doped glass coolers with other devices, it is desirable to develop luminescence coolers using semiconductors to facilitate their integration with semiconductor infrared sensors, low-noise electronic circuits, and computer chips.

Recently, several theoretical papers investigating the cooling characteristics of photoluminescence refrigeration have been published, ${ }^{3-5}$ including an analysis of photon recycling in a doped glass cube. ${ }^{5}$ However, the influence of photon recycling on cooling efficiency, cooling power density, and the conditions for cooling has not yet been discussed in detail for luminescence refrigeration in semiconductors. Furthermore, to date a simplified two-level model has been used that includes an assumption that all of the emitted photons trapped inside the semiconductor are recycled. ${ }^{3}$ Although such a model is valid for gases and doped glass, it does not predict many important characteristics of luminescence refrigeration in semiconductors, which have a continuous density of states, a large index of refraction, and a larger nonradiative recombination rate.

In this paper, an improved model that includes the rate equations for carriers and photons as well as spectral dependent photon recycling is used to study the characteristics of luminescence refrigeration in semiconductors. General expressions for cooling efficiency and cooling power density are presented, as well as the photon extraction factor and external quantum efficiency required to achieve luminescence refrigeration.

\section{THEORETICAL MODEL}

In semiconductors, the large index of refraction results in a small escape cone for luminescence and the large interband

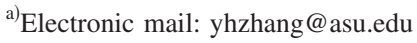

absorption coefficient results in a short mean free path for photons generated by radiative recombination. Consequently luminescence can undergo many absorption/emission cycles before escaping from the semiconductor. This process is commonly referred to as photon recycling ${ }^{6,7}$ and is not negligible in semiconductors with high spontaneous emission efficiencies, since the energy input through optical pumping or electrical injection can, on average, be recycled several times before escaping in the form of a photon or being lost to heat through nonradiative processes.

A schematic diagram of the optical processes in semiconductor photoluminescence refrigeration is shown in Fig. 1 , where laser light with photon energy $h \nu_{\text {in }}$ is absorbed via interband absorption $\alpha\left(h \nu_{\text {in }}, N\right)$. This process results in a photogenerated electron-hole pair density, of which fraction $\eta_{\text {iq }}$ recombines radiatively (emitting photons), and fraction $\left(1-\eta_{\text {iq }}\right)$ recombines nonradiatively (emitting phonons), heating the semiconductor. Of the emitted photons, some escape from the semiconductor, some are absorbed by free carriers (heating the semiconductor), and some are reabsorbed via interband absorption adding to the carrier density in the recycling process mentioned above. As the carrier density increases, interband absorption decreases, resulting in a steady state carrier density $N$ for a given pump density. As the pump density is increased, $N$ increases, and the interband absorption coefficient $\alpha\left(h \nu_{\text {in }}, N\right)$ decreases, eventually approaching zero under high excitation, thereby limiting the efficacy of further increases in pump density. A necessary, but not suf-

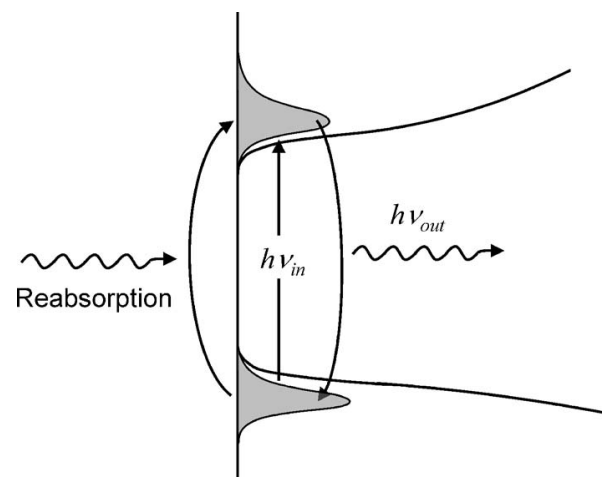

FIG. 1. Schematic diagram of the optical processes in optically pumped semiconductors, including photon recycling. 
ficient, condition for photoluminescence refrigeration is that the average photon energy of the emitted luminescence be greater than the photon energy of the pump laser, i.e., $h \bar{\nu}_{\text {out }}$ $-h \nu_{\text {in }}>0$. The optical processes in the electroluminescence refrigeration are similar to those in the photoluminescence refrigeration in semiconductors except that the carrier density is generated through electrical injection.

The rate equations used to quantify the processes described above are as follows ${ }^{8-10}$

$$
\begin{gathered}
\frac{d s(h \nu, N)}{d t}=r(h \nu, N) \frac{N}{\tau_{\mathrm{sp}}}-\alpha(h \nu, N) \frac{c}{n} s(h \nu, N) \\
-\alpha \frac{c}{n} s(h \nu, N)-\alpha \frac{c}{n} s(h \nu, N), \\
\frac{d N}{d t}=\alpha\left(h \nu_{\mathrm{in}}, N\right) \frac{c}{n} S_{\mathrm{in}}+\int \alpha(h \nu, N) \frac{c}{n} s(h \nu, N) d h \nu \\
-\frac{1}{\eta_{q}(N)} \frac{N}{\tau_{\mathrm{sp}}} .
\end{gathered}
$$

The second term on the right-hand side of both Eqs. (1) and (2) accounts for the spectral dependent photon recycling. $S_{\text {in }}$ is the pump photon density (number of photons per unit volume), $s(h \nu, N)$ is the luminescence photon spectral density (number of photons per unit volume per energy interval), $c$ is the speed of light in free space, $\tau_{\mathrm{sp}}$ is the spontaneous emission lifetime, $r(h \nu, N)$ is the normalized spontaneous emission rate per energy interval with $\int r(h \nu, N) d h \nu=1, \alpha(h \nu, N)$ is the interband absorption coefficient at $h \nu, h \nu_{\text {in }}$ is the pump photon energy, $\alpha_{i}$ is the internal loss due to free carrier and impurity absorption, and $\alpha_{e}$ is the average luminescence extraction loss over the photon path within the device, which depends on device geometry. $N$ is the total carrier density including the photoexcited carriers from both pumping and photon recycling. $\eta_{q}(N)$ is the spontaneous emission efficiency and is defined as

$$
\eta_{q}(N)=\frac{R_{\mathrm{sp}}(N)}{R_{\mathrm{sp}}(N)+R_{\mathrm{SRH}}(N)+R_{\text {Auger }}(N)},
$$

which depends on carrier density and material properties. $R_{\text {sp }}(N)=N / \tau_{\text {sp }}$ is the spontaneous emission rate, $R_{\mathrm{SRH}}$ is the Shockley-Read-Hall (SRH) recombination rate, and $R_{\text {Auger }}$ is the Auger recombination rate. The spontaneous emission efficiency in Eq. (3) is commonly used as the internal quantum efficiency, however, as discussed below, when photon recycling or stimulated emission are significant, the internal quantum efficiency is a function of Eq. (3). For electroluminescence refrigeration in light emitting diodes, the first term on the right-hand side of Eq. (2) changes to $J / q d$, where $J$ is the injection current density, $q$ is electron charge, and $d$ is the thickness of active region.

In the steady state, $d s / d t=d N / d t=0$, and the relationship between the photon density and the carrier density is

$$
s(h \nu, N)=\frac{r(h \nu, N)}{\left[\alpha(h \nu, N)+\alpha_{i}+\alpha_{e}\right](c / n)}\left(\frac{N}{\tau_{\mathrm{sp}}}\right) .
$$

Substituting Eq. (4) into Eq. (2), the absorbed laser power density is

$$
P_{\text {in }}=\alpha\left(h \nu_{\text {in }}, N\right) \frac{c}{n} S_{\text {in }} h \nu_{\text {in }}=\frac{N}{\tau_{\text {sp }}}\left[\frac{1}{\eta_{q}(N)}-\gamma_{r}(N)\right] h \nu_{\text {in }},
$$

where $\gamma_{r}(N)$ is the photon recycling factor, ${ }^{8,9}$ with

$$
\gamma_{r}(N)=\int \frac{r(h \nu, N) \alpha(h \nu, N)}{\alpha(h \nu, N)+\alpha_{i}+\alpha_{e}} d h \nu,
$$

which is defined as the fraction of the spontaneously emitted photons that are reabsorbed in the active region. ${ }^{7-9}$ Unlike the overlap integral of the luminescence and absorption spectra $\int r_{\mathrm{sp}}(h \nu, N) \alpha(h \nu, N) d h \nu, \gamma_{r}(N)$ is a dimensionless parameter with $0 \leqslant \gamma_{r}(N) \leqslant 1$ when $\alpha(h \nu, N) \geqslant 0$. Similarly, the steady state luminescence output power density is given by

$$
\begin{aligned}
P_{\text {out }} & =\int \alpha_{e} s(h \nu, N) \frac{c}{n} h \nu d h \nu \\
& =\frac{N}{\tau_{\mathrm{sp}}} \int \gamma_{\mathrm{se}}(h \nu, N) r(h \nu, N) h \nu d h \nu,
\end{aligned}
$$

where $\quad \gamma_{\mathrm{se}}(h \nu, N)=\alpha_{e} /\left[\alpha(h \nu, N)+\alpha_{i}+\alpha_{e}\right], \quad 0 \leqslant \gamma_{\mathrm{se}}(h \nu, N)$ $\leqslant \alpha_{e} /\left(\alpha_{e}+\alpha_{i}\right)$ when $\alpha(h \nu, N) \geqslant 0$, is the spectral photon extraction factor, from which the photon extraction factor is given by

$$
\gamma_{e}=\int r(h \nu, N) \gamma_{\mathrm{se}}(h \nu, N) d h \nu,
$$

and is defined as the ratio of the number of photons emitted into free space to the number of photons spontaneously generated in the active region. The photon extraction factor can be greater than unity when $\alpha(h \nu, N)<0$, due to the intensification of photon extraction by simulated emission.

Via conservation of energy, the cooling efficiency $\eta_{c}$ and the cooling power density $P_{c}$ for photoluminescence refrigeration in semiconductors are, respectively,

$$
\begin{aligned}
\eta_{c}\left(h \nu_{\mathrm{in}}, N\right)= & \frac{P_{\mathrm{out}}-P_{\mathrm{in}}}{P_{\text {in }}}=\int r(h \nu, N) \\
& \times\left[\frac{\eta_{q}(N) \gamma_{\mathrm{se}}(h \nu, N)}{1-\eta_{q}(N) \gamma_{r}(N)} \frac{h \nu}{h \nu_{\mathrm{in}}}-1\right] d h \nu
\end{aligned}
$$

and

$$
\begin{aligned}
P_{c}\left(h \nu_{\text {in }}, N\right)= & P_{\text {out }}-P_{\text {in }} \\
= & \frac{N}{\tau_{\text {sp }}}\left\{\int \gamma_{\text {se }}(h \nu, N) r(h \nu, N) h \nu d h \nu\right. \\
& \left.-\left[\frac{1}{\eta_{q}(N)}-\gamma_{r}(N)\right] h \nu_{\text {in }}\right\} .
\end{aligned}
$$

To realize net cooling, the total power density of emitted photoluminescence must be higher than that of the absorbed pump laser light, namely, $\eta_{c}>0$, which from Eq. (9) results in

$$
\int \frac{h \nu}{h \nu_{\mathrm{in}}} r(h \nu, N) \frac{\eta_{q}(N) \gamma_{\mathrm{se}}(h \nu, N)}{1-\eta_{q}(N) \gamma_{r}(N)} d h \nu>1 .
$$

In addition to the fundamental requirement that $\int\left(h \nu / h \nu_{\text {in }}\right) r(h \nu, N) d h \nu=h \bar{\nu}_{\text {out }} / h \nu_{\text {in }}>1$ for cooling, sufficiently high spontaneous emission efficiency and photon ex- 
TABLE I. The definitions for the parameters used in this paper.

\begin{tabular}{|c|c|c|}
\hline Parameter & Symbol & Definition \\
\hline Spontaneous emission efficiency & $\eta_{q}$ & $\begin{array}{l}\text { photons spontaneously generated in active region } \\
\text { carriers that spontaneously and nonradiatively recombine in active region } \\
\qquad \eta_{q}(N)=\frac{R_{\mathrm{sp}}(N)}{R_{\mathrm{sp}}(N)+R_{\mathrm{SRH}}(N)+R_{\text {Auger }}(N)}\end{array}$ \\
\hline Photon extraction factor & $\gamma_{e}$ & $\begin{array}{c}\frac{\text { photons emitted into free space }}{\text { photons spontaneously generated in active region }} \\
\gamma_{e}=\int \frac{r(h \nu, N) \alpha_{e}}{\alpha(h \nu, N)+\alpha_{e}+\alpha_{i}} d h \nu\end{array}$ \\
\hline Photon recycling factor & $\gamma_{r}$ & $\begin{array}{c}\frac{\text { photons reabsorbed in active region }}{\text { photons spontaneously generated in active region }} \\
\gamma_{r}=\int \frac{r(h \nu, N) \alpha(h \nu, N)}{\alpha(h \nu, N)+\alpha_{e}+\alpha_{i}} d h \nu\end{array}$ \\
\hline Internal quantum efficiency & $\eta_{\text {iq }}$ & $\begin{array}{c}\frac{\text { net photons generated in active region }}{\text { carriers injected into active region }}, \\
\eta_{\mathrm{iq}}(N)=\frac{R_{\mathrm{sp}}(N)-\int \alpha(h \nu, N)(c / n) s(h \nu, N) d h \nu}{R_{\mathrm{sp}}(N)+R_{\mathrm{SRH}}(N)+R_{\text {Auger }}(N)-\int \alpha(h \nu, N)(c / n) s(h \nu, N) d h \nu} \\
=\frac{\left(1-\gamma_{r}\right) \eta_{q}}{1-\gamma_{r} \eta_{q}}\end{array}$ \\
\hline Extraction efficiency & $\eta_{e}$ & $\frac{\text { photons emitted into free space }}{\text { net photons generated in active region }}, \eta_{e}=\frac{\alpha_{e}}{\alpha_{e}+\alpha_{i}}=\frac{\gamma_{e}}{1-\gamma_{r}}$ \\
\hline External quantum efficiency & $\eta_{\mathrm{eq}}$ & $\frac{\text { photons emitted into free space }}{\text { carriers injected into active region }}, \eta_{\mathrm{eq}}=\frac{\eta_{q} \gamma_{e}}{1-\eta_{q} \gamma_{r}}=\eta_{\mathrm{iq}} \eta_{e}$ \\
\hline
\end{tabular}

traction are also necessary. Equations (9)-(11) give the general expressions for the cooling efficiency, cooling power density, and the cooling condition for photoluminescence refrigeration in semiconductors and are applicable to electroluminescence refrigeration when $h \nu_{\text {in }}$ is replaced by the injection bias multiplied by the electron charge. In the present paper, heating due to the parasitic absorption of pump light in photoluminescence refrigeration or Joule heating caused by series resistance in electroluminescence refrigeration is neglected, which, however, can be substantial when pumping power is large or electrical injection is high.

Table I lists the definitions of the parameters used in this paper. The internal quantum efficiency is related to the spontaneous emission efficiency by $\eta_{\text {iq }}=\left(1-\gamma_{r}\right) \eta_{q} /\left(1-\gamma_{r} \eta_{q}\right)$ and the extraction efficiency is related to the photon extraction factor by $\eta_{e}=\gamma_{e} /\left(1-\gamma_{r}\right)$, which results in an external quantum efficiency that is $\eta_{\mathrm{eq}}=\eta_{\mathrm{iq}} \eta_{e}=\eta_{q} \gamma_{e} /\left(1-\eta_{q} \gamma_{r}\right)$. The term $\int \alpha(h \nu, N)(c / n) s(h \nu, N) d h \nu$ in the definition of internal quantum efficiency accounts for the influence of photon recycling when $\alpha(h \nu, N)>0$ and the influence stimulated emission when $\alpha(h \nu, N)<0$. The internal quantum efficiency depends not only on carrier density and material properties but also on internal loss, refractive index, and device geometry.

\section{RESULTS AND DISCUSSIONS}

Under low injection, the absorption of recycled photons is expected to be uniform across the linewidth $(\sim k T)$ of the spontaneous emission spectrum; this results in a spectral photon extraction factor $\gamma_{\mathrm{se}}(h \nu, N)$ that is weakly dependent on photon energy. In this case Eqs. (9)-(11) are simplified by assuming that the photon extraction factor and interband absorption coefficient are constant over the integration, in which case the cooling efficiency under low injection becomes [see Eq. (9)]

$$
\begin{aligned}
\eta_{c} & \approx \frac{\eta_{q} \gamma_{e}\left(\eta_{\mathrm{ic}}+1\right)}{1-\eta_{q} \gamma_{r}}-1=\frac{\eta_{q} \gamma_{e}\left(\eta_{\mathrm{ic}}+1\right)}{1-\eta_{q}\left(1-\gamma_{e}\right)\left(1+\alpha_{i} / \bar{\alpha}\right)^{-1}}-1 \\
& =\eta_{\mathrm{eq}}\left(\eta_{\mathrm{ic}}+1\right)-1,
\end{aligned}
$$

where $\bar{\alpha}=\int r(h \nu, N) \alpha(h \nu, N) d h \nu$. The ideal cooling efficiency is defined as

$$
\eta_{\text {ic }}=\int r(h \nu, N)\left(h \nu / h \nu_{\text {in }}-1\right) d h \nu=h \bar{\nu}_{\text {out }} / h \nu_{\text {in }}-1,
$$

which is achieved when the external quantum efficiency is unity. The ideal cooling efficiency is positive (a necessary but not sufficient condition for cooling) when $h \nu_{\text {in }}<h \bar{\nu}_{\text {out }}$ $\approx\left(E_{g}+k T\right)$, where $E_{g}$ is the band gap energy.

The minimum external quantum efficiency required to access cooling is [see Eq. (12)]

$$
\eta_{\mathrm{eq}}^{\min }=1 /\left(1+\eta_{\mathrm{ic}}\right) \text {. }
$$

The minimum external quantum efficiency $\eta_{\mathrm{eq}}^{\mathrm{min}}$ is plotted in Fig. 2 against the ideal cooling efficiency $\eta_{\text {ic }}$. In this plot, the pump energy is assumed to be at the band gap energy ( $h \nu_{\text {in }}$ $=E_{g}$ ) and $h \bar{\nu}_{\text {out }}-h \nu_{\text {in }}=k T$ is chosen as a reasonable value for low injection, resulting in an ideal cooling efficiency $\eta_{\text {ic }}$ $=k T / E_{g}$. The shaded area labeled "cooling region" shows the region where luminescence refrigeration is accessible and the solid curve is the $\eta_{c}=0$ boundary. Cooling is only accessible when the external quantum efficiency is greater than $\eta_{\mathrm{eq}}^{\mathrm{min}}$. To 


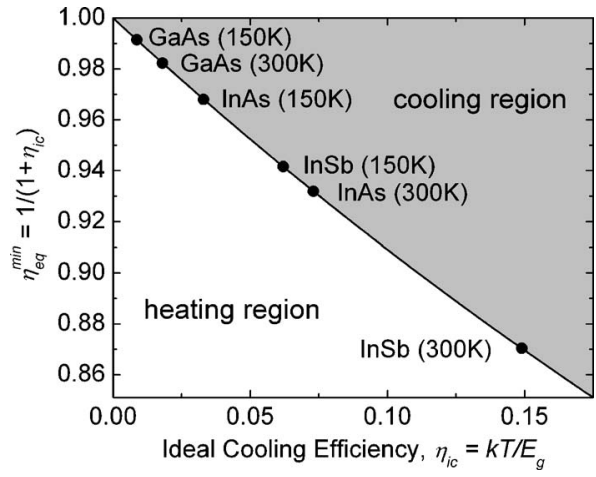

FIG. 2. Minimum external quantum efficiency $\left(\eta_{\mathrm{eq}}^{\mathrm{min}}\right)$ vs ideal cooling efficiency $\left(\eta_{\mathrm{ic}}=k T / E_{g}\right)$. The cooling efficiency is positive in the shaded area and zero along the solid line.

put the accessibility of cooling in perspective with common optoelectronic semiconductors, the ideal cooling efficiencies $\left(\eta_{\text {ic }} \approx k T / E_{g}\right)$ are shown as solid circles for the band gap energies of GaAs, InAs, and InSb at two temperatures, 150 and $300 \mathrm{~K}$.

In the ideal spontaneous emission efficiency limit $\left(\eta_{q}\right.$ $=1$ ), the minimum photon extraction factor required to access cooling is [see Eqs. (6), (8), and (12)]

$$
\gamma_{e}^{\min }=\frac{1}{1+\left(1+\bar{\alpha} / \alpha_{i}\right) \eta_{\text {ic }}} .
$$

The minimum photon extraction factor $\gamma_{e}^{\min }$ versus ideal cooling efficiency $\eta_{\text {ic }}=k T / E_{g}$ is shown in Fig. 3 for several values of $\bar{\alpha} / \alpha_{i}$. The solid curves give the $\eta_{c}=0$ boundary for each $\bar{\alpha} / \alpha_{i}$ value, and the $\eta_{c}>0$ cooling regions are again located above each solid curve. The ideal cooling efficiencies $\left(\eta_{\mathrm{ic}} \approx k T / E_{g}\right)$ are shown as vertical dotted lines for GaAs, InAs, and InSb band gap energies for temperatures of 150 and $300 \mathrm{~K}$. The minimum photon extraction factor required to access cooling increases as $\bar{\alpha} / \alpha_{i}$ decreases. The $\bar{\alpha} / \alpha_{i}=0$ curve is identical to the curve shown in Fig. 2, since $\gamma_{e}^{\mathrm{min}}$ $=1 /\left(1+\eta_{\mathrm{ic}}\right)=\eta_{\mathrm{eq}}^{\min }$ when $\bar{\alpha} / \alpha_{i}=0$.

In the ideal extraction limit $\left(\alpha_{i}=0\right.$ and $\left.\eta_{e}=1\right)$, the minimum spontaneous emission efficiency required to access cooling is [see Eqs. (6), (8), and (12)]

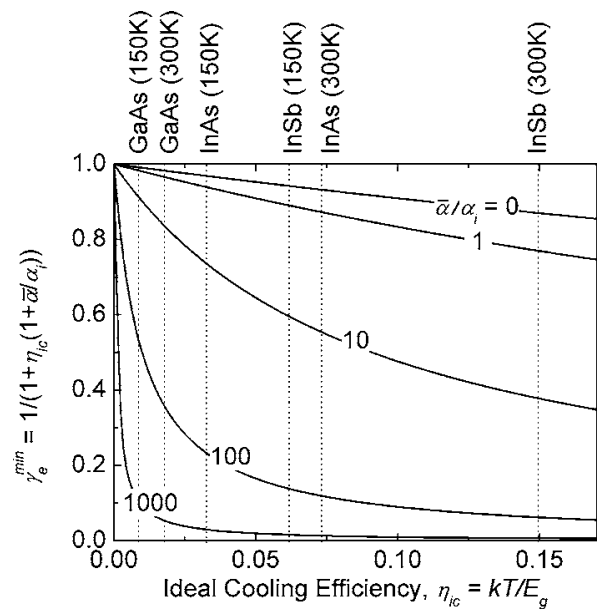

FIG. 3. Minimum photon extraction factor $\left(\gamma_{e}^{\mathrm{min}}\right)$ vs ideal cooling efficiency $\left(\eta_{\text {ic }}=k T / E_{g}\right)$ for various values of $\bar{\alpha} / \alpha_{i}$.

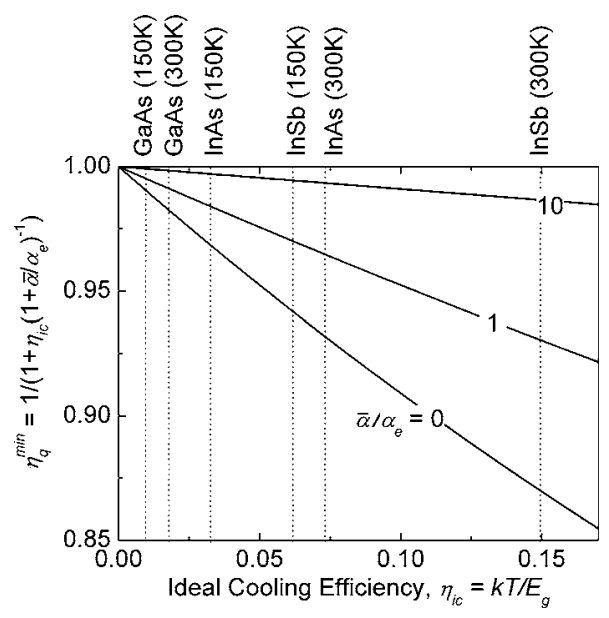

FIG. 4. Minimum spontaneous emission efficiency $\left(\eta_{q}^{\mathrm{min}}\right)$ vs ideal cooling efficiency $\left(\eta_{\mathrm{ic}}=k T / E_{g}\right)$ for various values of $\bar{\alpha} / \alpha_{e}$.

$$
\eta_{q}^{\min }=\frac{1}{1+\left(1+\bar{\alpha} / \alpha_{e}\right)^{-1} \eta_{\mathrm{ic}}} .
$$

The minimum spontaneous emission efficiency $\eta_{q}^{\min }$ versus ideal cooling efficiency $\eta_{\mathrm{ic}}=k T / E_{g}$ is shown in Fig. 4 for several values of $\bar{\alpha} / \alpha_{e}$. Similarly, the solid curves give the $\eta_{c}=0$ boundary for each $\bar{\alpha} / \alpha_{e}$ value, and the $\eta_{c}>0$ cooling regions are again located above each solid curve. The ideal cooling efficiencies $\left(\eta_{\mathrm{ic}} \approx k T / E_{g}\right)$ are shown as vertical dotted lines for GaAs, InAs, and InSb band gap energies for temperatures of 150 and $300 \mathrm{~K}$. The minimum spontaneous emission efficiency required to access cooling decreases as $\bar{\alpha} / \alpha_{e}$ decreases. The $\bar{\alpha} / \alpha_{e}=0$ curve is identical to the curve shown in Fig. 2 , since $\eta_{q}^{\mathrm{min}}=1 /\left(1+\eta_{\mathrm{ic}}\right)=\eta_{\mathrm{eq}}^{\min }$ when $\bar{\alpha} / \alpha_{e}$ $=0$.

Consider the two extreme cases where the recycling of trapped photons is (i) a minimum $\left(\bar{\alpha} \ll \alpha_{i}\right)$ or (ii) a maximum $\left(\bar{\alpha} \gg \alpha_{i}\right)$. In the minimum photon recycling case, the band to band absorption coefficient at the luminescence wavelengths is much smaller than the internal loss, resulting in the conversion of trapped luminescence into heat through internal absorption losses. In which case $\eta_{q} \approx \eta_{\mathrm{iq}}$ and $\gamma_{e} \approx \eta_{e}$, the resulting cooling efficiency becomes

$$
\eta_{c} \approx \eta_{q} \gamma_{e}\left(\eta_{\mathrm{ic}}+1\right)-1,
$$

and the resulting minimum photon extraction factor becomes $\gamma_{e}^{\mathrm{min}}=1 /\left(1+\eta_{\mathrm{ic}}\right)$. Equation (17) gives the same result as reported in a previous work on electroluminescence refrigeration. ${ }^{11}$

In the maximum photon recycling case, the band to band absorption coefficient at the luminescence wavelengths is much greater than the internal loss and the trapped luminescence is reabsorbed in the active region, generating additional carriers that also contribute to cooling, resulting in a cooling efficiency that becomes

$$
\eta_{c} \approx \frac{\eta_{q} \gamma_{e}\left(\eta_{\mathrm{ic}}+1\right)}{1-\eta_{q}\left(1-\gamma_{e}\right)}-1,
$$

and a minimum extraction efficiency that becomes $\gamma_{e}^{\min }$ $\approx 1 /\left(1+\eta_{\mathrm{ic}} \bar{\alpha} / \alpha_{i}\right)$. Equation (18) is similar to the cooling ef- 


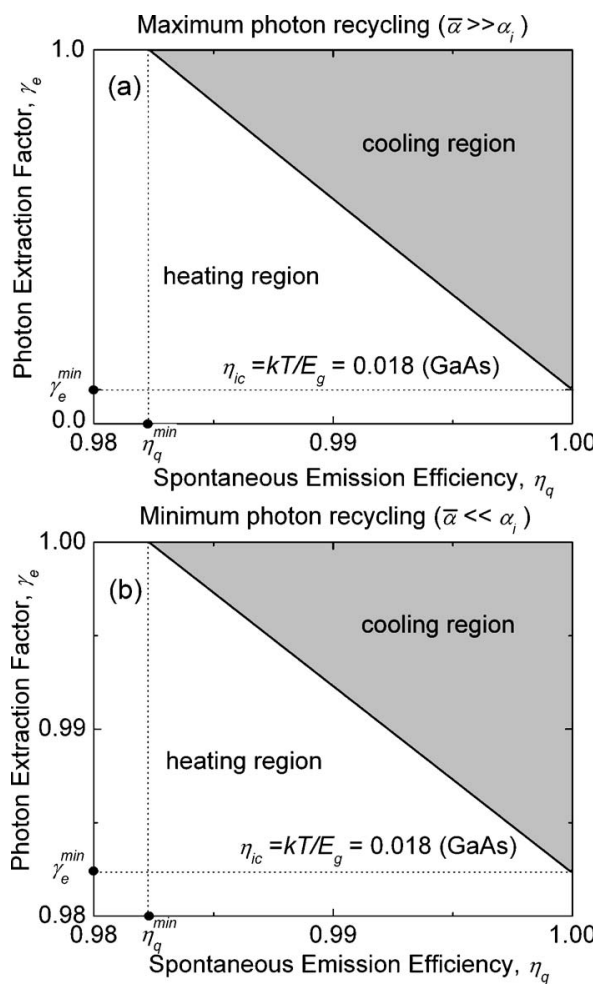

FIG. 5. Photon extraction factor vs spontaneous emission efficiency for the maximum photon recycling case in (a) and for the minimum photon recycling case in (b). The cooling efficiency is positive in the shaded area and zero along the solid line. The ideal cooling coefficient $\left(\eta_{\mathrm{ic}}=k T / E_{g}\right)$ used is for the GaAs band gap energy.

ficiency for semiconductor laser cooling discussed by SheikBahae and Epstein. ${ }^{3}$

The cooling efficiency as a function of spontaneous emission efficiency and photon extraction factor is shown in Fig. 5 for the GaAs band gap energy, which has an ideal cooling efficiency of $\eta_{\text {ic }}=k T / E_{g}=0.018$ at room temperature. The shaded areas show the cooling regions and the solid curves give the $\eta_{c}=0$ boundaries between the cooling and heating regions. As shown in Fig. 5, cooling is only accessible when the spontaneous emission efficiency is greater than $\eta_{q}^{\text {min }}$ which is 0.982 for the GaAs band gap energy. Furthermore, the cooling efficiency $\eta_{c}$ increases monotonically from the $\eta_{c}=0$ boundary toward the upper right corner of the cooling region where it reaches its maximum value $\eta_{\text {ic }}$ at $\eta_{q}=\gamma_{e}=1$. When $\eta_{q}=1$ and $\bar{\alpha} \ll \alpha_{i}$ (minimum photon recycling), cooling is only accessible if the photon extraction factor is greater than $\gamma_{e}^{\mathrm{min}}=1 /\left(1+\eta_{\mathrm{ic}}\right)$, which is 0.982 for the GaAs band gap energy. When $\eta_{q}=1$ and $\bar{\alpha} \gg \alpha_{i}$ (maximum photon recycling), cooling is only accessible if the extraction efficiency is greater than $\gamma_{e}^{\min } \approx 1 /\left(1+\eta_{\text {ic }} \bar{\alpha} / \alpha_{i}\right)$, which is 0.05 for the GaAs band gap energy when $\bar{\alpha} / \alpha_{i}=1000$.

It appears that smaller band gap materials and higher temperatures make cooling more accessible. However, it is worth noting that both the spontaneous emission efficiency and the photon extraction factor decrease as the band gap energy decreases and/or the temperature increases. For example, the spontaneous emission efficiency is lower in small band gap materials due to a smaller spontaneous emission coefficient and a larger Auger recombination coefficient that also strongly increases with temperature. Moreover, the photon extraction factor is lower for long wavelength luminescence due to larger internal losses caused by parasitic absorption (owing to free carriers and impurities) which also increases with temperature. The choice of band gap for luminescence cooling is a trade-off between ideal cooling efficiency, which increases as the band gap narrows and/or temperature increases, spontaneous emission efficiency, and the photon extraction factor that typically decrease as the band gap narrows and/or temperature increases. A more detailed analysis of the influence of band gap energy on the cooling characteristics is beyond the scope of this paper and will be published separately.

\section{CONCLUSIONS}

In conclusion, the cooling efficiency, cooling power density, and limitations of semiconductor luminescence refrigeration are investigated using a rate equation model that includes the role of spectral dependent photon recycling. The boundaries for luminescence refrigeration are defined for the case where the emission linewidth is narrow $(\sim k T)$. The analysis for the two extreme photon recycling cases shows that photon recycling significantly contributes to luminescence refrigeration when the luminescence extraction is small.

\section{ACKNOWLEDGMENT}

This work is supported by a MURI program from the Air Force Office of Scientific Research, Grant No. FA9550-041-0374.

${ }^{1}$ R. I. Epstein, M. I. Buchwald, B. C. Edwards, T. R. Gosnell, and C. E. Mungan, Nature (London) 377, 500 (1995).

${ }^{2}$ C. W. Hoyt, M. Sheik-Bahae, R. I. Epstein, B. Edward, and J. Anderson, Phys. Rev. Lett. 85, 3600 (2000).

${ }^{3}$ M. Sheik-Bahae and R. I. Epstein, Phys. Rev. Lett. 92, 247403 (2004).

${ }^{4}$ D. H. Huang, T. Apostolova, P. M. Alsing, and D. A. Cardimona, Phys. Rev. B 70, 033203 (2004).

${ }^{5}$ B. Heeg, P. A. DeBarber, and G. Rumbles, Appl. Opt. 44, 3117 (2005).

${ }^{6}$ F. Stern and J. M. Woodall, J. Appl. Phys. 45, 3904 (1974).

${ }^{7}$ P. Asbeck, J. Appl. Phys. 48, 820 (1977).

${ }^{8}$ T. Tamanuki, F. Koyama, and K. Iga, Jpn. J. Appl. Phys., Part 1 31, 1810 (1992).

${ }^{9}$ T. Baba, R. Watanabe, K. Asano, F. Koyama, and K. Iga, Jpn. J. Appl. Phys., Part 1 35, 97 (1996).

${ }^{10}$ S.-L. Chuang, Physics of Optoelectronic Devices (Wiley, New York, 1995).

${ }^{11}$ J.-B. Wang, D. Ding, S.-Q. Yu, S. R. Johnson, and Y.-H. Zhang, Technical Digest of CLEO/QELS 2005, 2005 (unpublished), p. QThI7. 